Basáñez Muñoz, A. de J., Jordán Garza, A. G., Serrano, A., Capistran Barradas, A., Naval Ávila, C., \& Cuervo López, L. (2021). Estructura poblacional del árbol Avicennia germinans (Acanthaceae) en un manglar alterado del Golfo de México. Revista de Biología Tropical, 69(4), 12641275. https://doi.org/10.15517/rbt.v69i4.47353

\title{
Estructura poblacional del árbol Avicennia germinans (Acanthaceae) en un manglar alterado del Golfo de México
}

Agustín de J. Basáñez Muñoz ${ }^{*}$; (D) https://orcid.org/0000-0002-6063-7850

Adán G. Jordán Garza ${ }^{1}$; (iD https://orcid.org/0000-0002-9856-2276

Arturo Serrano2; (D) https://orcid.org/0000-0002-0175-458X

Ascención Capistran Barradas ${ }^{1}$; (D) https://orcid.org/0000-0002-1403-1575

Celina Naval Ávila1; iD https://orcid.org/0000-0001-7638-4918

Liliana Cuervo López ${ }^{2}$; (D) https://orcid.org/0000-0002-3661-6355

1. Maestría en Manejo de Ecosistemas Marinos y Costeros, Universidad Veracruzana, Tuxpan, Veracruz, México; abasanez@uv.mx (*Correspondencia), ajordan@uv.mx, acapistran@uv.mx, cnaval@uv.mx

2. Cuerpo Académico Manejo de Ambientes Marinos y Costeros, Universidad Veracruzana, Tuxpan, Veracruz, México; arserrano@uv.mx, lcuervo@uv.mx

Recibido 10-VI-2021. Corregido 14-X-2021. Aceptado 24-XI-2021.

\section{ABSTRACT \\ Population structure of Avicennia germinans trees (Acanthaceae) in a disturbed mangrove in the Gulf of Mexico}

Introduction: Globally, mangroves are declining; characterizing their structure and regeneration at different disturbance levels can help understand their responses to stressful situations.

Objective: The study's primary goal was to analyze the mangrove community structure and to estimate the populations' trajectories of Avicenia germinans at different mangrove sites that present three levels of disturbance. Methods: Eight transects of approximately $500 \mathrm{~m}$ in length and laid perpendicular to the Tamapamchoco lagoon, Veracruz, intersected three levels of disturbance currently present on the mangrove forest. On each transect, $10 \times 10 \mathrm{~m}, 5 \times 5 \mathrm{~m}$, and $1 \times 1 \mathrm{~m}$ quadrants served to account for all mangrove trees by species and stage of maturity in 2017 and 2018.

Results: The most abundant species was: A. germinans followed by Rhizophora mangle and Laguncularia racemosa. Based on their abundance, we found significant differences for the three levels of disturbance (ANOSIM $\mathrm{R}=0.6, \mathrm{P}<0.001$ ) presented in a non-metric ordination (nMDS). A life table for $A$. germinans allowed a population analysis that showed an intrinsic rate of population increase (r) that was negative at the disturbed sites (-0.0027 and -0.0774$)$ and positive (0.0289) at the apparently undisturbed site. Seedling to juvenile stage survival ranged from $50 \%$ at the undisturbed site to 5 and $4 \%$ at the disturbed sites.

Conclusions: Measures to increase the survival of seedlings are necessary to reverse the decreasing population trajectories at the disturbed sites; otherwise, the mangrove will have reduced viability in the medium term.

Key words: mangrove; fragmentation; perturbation; life tables; population dynamics; size classes, disturbance. 
Los bosques de mangle son ecosistemas diversos que proveen a las comunidades aledañas de una gran variedad de bienes y servicios (Ewel et al., 1998; Owuor et al., 2019). En la actualidad estos ecosistemas están amenazados por cambios a nivel global, como el aumento de la temperatura y del nivel medio del mar (Chen et al., 2011; Osland et al., 2017; Sasmito et al., 2015). Pero también por efectos a nivel local, como lo son el cambio de usos de suelo en la zona costera, que desertifican los bosques de mangle para dar lugar a construcciones humanas (Chow 2017; Hamilton \& Casey, 2016). En México, los manglares son uno de los ecosistemas costeros más importantes en la región del Golfo de México. Los estados de Tamaulipas, Veracruz y Tabasco presentaban en 2015 un total de 87048 ha (ValderramaLanderos et al., 2017), estando conformados por cuatro especies: Rhizophora mangle L., Avicennia germinans (L.) L., Laguncularia racemosa (L.) C.F. Gaertn. y Conocarpus erectus L. (Moreno-Casasola et al., 2016). En Veracruz, la región de la cuenca hidrológica, que comprende los ríos Tuxpan y Nautla, cuenta con un gran cantidad de manglares con superficies menores de 1000 ha, distribuidas en los sistemas lagunares de Tamiahua, Tampamachoco y San Agustín (Moreno et al., 2002). De estos sistemas, la Laguna de Tampamachoco, junto con sus manglares asociados, están comprendidos en el Sitio Ramsar No. 1602 "Manglares y humedales de Tuxpan" con una extensión de 6870 ha, de las cuales más de 3 000 ha son de manglar. El sitio fue decretado en 2006 (Comisión Nacional de Áreas Naturales Protegidas [CONANP], 2019).

Los manglares del Sitio Ramsar No. 1602 han sido estudiados con relación a su composición, estructura, productividad, fenología, usos, restauración y captura de carbono, pero sobre todo por presentar un área con signos de alteración en dónde se han perdido varias hectáreas de mangle (Basáñez-Muñoz et al., 2006; Basáñez-Muñoz et al., 2008; Basáñez-Muñoz et al. 2016; López-Portillo et al., 2014; Vovides et al., 2011). En el área de estudio, Vovides et al. (2011) atribuyen la mortalidad al cambio gradual de fijación de nitrógeno de sitios no alterados a sitios alterados, mencionando que las bacterias fijadoras de nitrógeno son sensibles al incremento de salinidad, $\mathrm{pH}$ y contenido de amonio del suelo. Estos autores mencionan que el manglar presenta deterioro desde 1980 y le atribuyen la principal causa, a la construcción de tres terraplenes que soportan torres de líneas de transmisión del Complejo Termoeléctrico "Presidente Adolfo López Mateos". Esta construcción ocasionó la interrupción del flujo hidrológico de sur a norte, ocasionando una hipersalinización (120 ups) del suelo que originó el deterioro de más de 20 ha de manglar. López-Portillo et al. (2014) establecen que la muerte comienza de las ramas altas de individuos hacia las ramas bajas. Este tipo de mortalidad es atribuible a una enfermedad conocida como "top dying" (Awal, 2014a; Awal, 2014b; Giri et al., 2014; Islam \& Bhulyan, 2018; Islam et al., 2019; Rahman, 1994). Para conocer los procesos de degradación sobre el manglar, producto de la alteración, se planteó como objetivo analizar la relación entre diferentes sitios que presentan alteración y proyectar la dinámica poblacional de $A$. germinans en función de estadios poblacionales en los distintos sitios de alteración.

\section{MATERIALES Y MÉTODOS}

Área de estudio: El área de trabajo se encuentra al norte del estado de Veracruz, a 13 kilómetros al oeste de la ciudad y puerto de Tuxpan. Es en un bosque de mangle, situado frente a la entrada del Complejo Termoeléctrico "Presidente Adolfo López Mateos", teniendo en su límite oeste la Laguna de Tampamachoco. Los manglares relacionados con esta laguna se encuentran en el poligonal norte del Sitio Ramsar No. 1602 "Manglares y Humedales de Tuxpan". El sistema lagunar de Tampamachoco, se ubica entre los paralelos

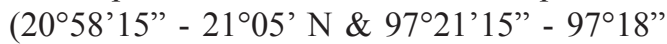
W) (CONANP, 2019) (Fig. 1).

El área de estudio comienza a orillas de la Laguna de Tampamachoco y termina a un lado de la construcción de la central termoeléctrica. 

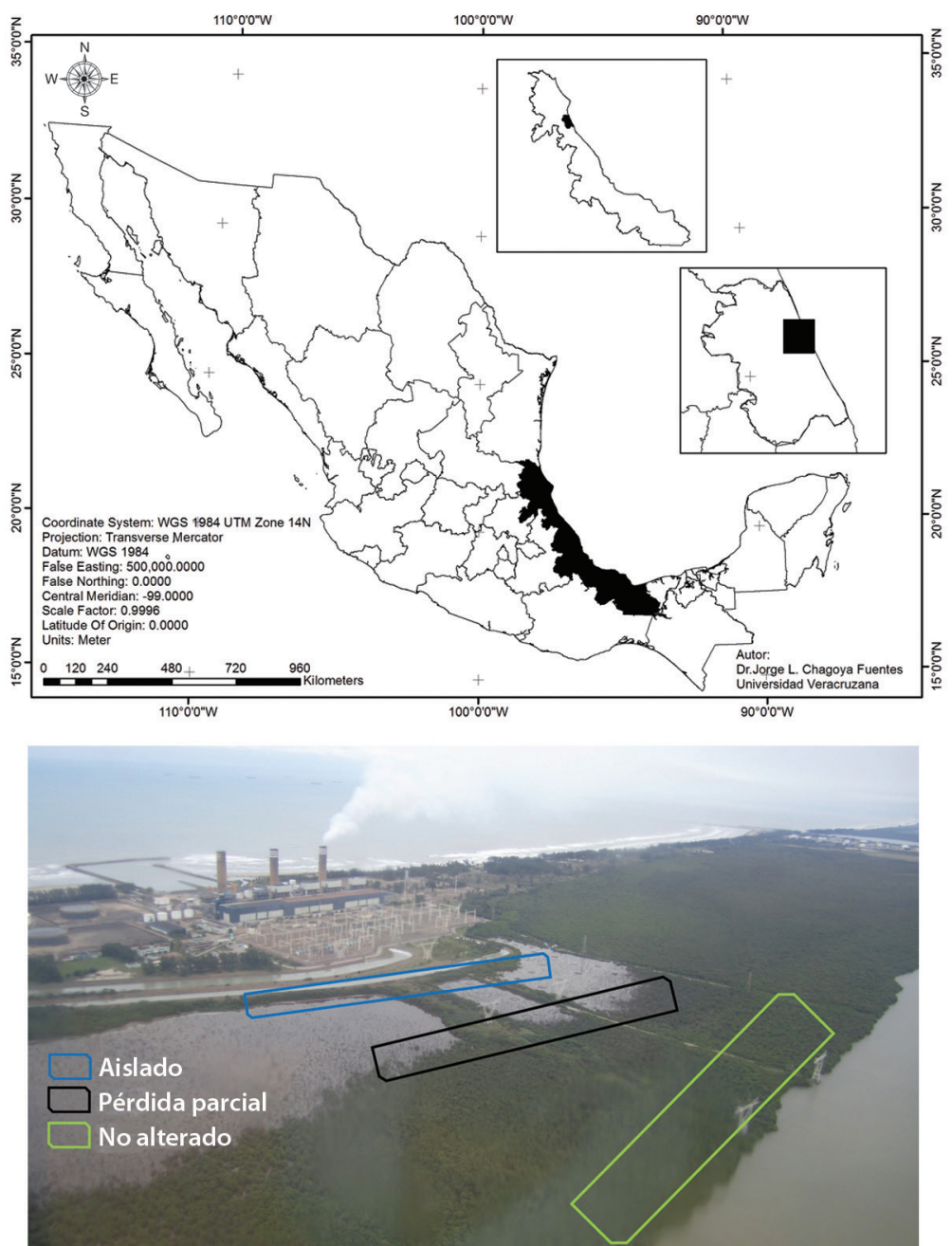

Fig. 1. Área de estudio con los tipos de alteración, cuadros de colores (verde $=$ sin alteración, negro $=$ alterado y azul $=$ aislado), los transectos y los fragmentos del bosque (Z I, Z II y Z III).

Fig. 1. Study area showing the types of perturbation, color squares (green= no perturbation, black $=$ perturbed and blue $=$ isolated), the transects and forest fragments (ZI, ZII and ZIII).

A partir de la central se construyeron tres terraplenes que dividen el área en tres fragmentos: el primer fragmento (poligonal Z I-sur) tiene una extensión de 15.47 ha, el segundo (poligonal Z II-centro) presenta 5.48 ha y el último fragmento (poligonal Z III-norte), que es el más extenso, tiene 90.7 ha. El bosque de manglar puede dividirse en áreas de distintos niveles de alteración, determinadas a priori por cambios visibles en la estructura del manglar (Vovides et al., 2011). En los primeros $200 \mathrm{~m}$ de la orilla, hacia el interior del bosque, se presenta un área aparentemente sin daño (no alterado), posteriormente hay $100 \mathrm{~m}$ de bosque con pérdida parcial de árboles, con evidencia de estrés en los árboles determinada por muerte total o parcial de su copa (alterado), para continuar con $200 \mathrm{~m}$ de una zona completamente degradada (todos los árboles muertos) y, finalmente, una franja de $\pm 10 \mathrm{~m}$ de un manglar que se 
desarrolló aprovechando el talud formado en la construcción de un canal de descarga de agua del complejo termoeléctrico (aislado), separado del área completamente degradada debido a la elevación topográfica (Fig. 1).

En 2017, se realizaron ocho transectos perpendiculares a la laguna, atravesando todos los gradientes del estado del bosque. Los individuos de mangle de las tres especies reportadas (A. germinans, $R$. mangle y L. racemosa) fueron contados y clasificados en seis estadios según su tamaño: tres estadios de regeneración (Kairo et al., 2002) y tres de estructura forestal (Cintrón-Molero \& Schaeffer-Novelli, 1992). A lo largo de cada transecto, los conteos se realizaron dentro de cuatro cuadrantes de $10 \times 10$ $\mathrm{m}$ (para árboles grandes) y dentro de éstos, uno de 5 x $5 \mathrm{~m}$, y a su vez, de 1 x $1 \mathrm{~m}$ (para arboles pequeños y plántulas). Las diferencias en las clases de regeneración se basaron en la altura, $<40 \mathrm{~cm}$ (plántulas), $40 \mathrm{a}<150 \mathrm{~cm}$ (jóvenes) y $150 \mathrm{a}<300 \mathrm{~cm}$, pero $<2.5 \mathrm{~cm}$ diámetro a la altura del pecho (árbol joven "a"), y las clases de estructura en función al diámetro a la altura del pecho (DAP), $2.5 \mathrm{a}<5 \mathrm{~cm}$ (árbol joven "b"), $5 \mathrm{a}<10 \mathrm{~cm}$ (adulto) y $>10 \mathrm{~cm}$ (maduro). El inicio de la fase reproductiva se consideró a partir de la clase árbol joven "b", con una edad promedio de cinco años (Clarke, 1995). Los individuos $>2.5 \mathrm{~cm}$ en el área alterada fueron medidos independientemente de su condición de estrés presente (es decir, con observación manifiesta de mortalidad de las partes altas de su copa o no).

Análisis de datos: Una matriz de las abundancias de las tres especies de mangle por estadios (regeneración y estructura), por transecto, poligonal y zona afectada de manglar, se empleó en un análisis de ordenación multidimensional no métrico (Warwick \& Clarke, 1991). Utilizando los niveles de afectación como factor, se realizó un análisis de similitudes y un análisis de similitudes porcentuales para conocer si hay diferencias significativas entre niveles de alteración, y qué especies por estadio contribuyen a dichas diferencias (Clarke \& Warwick, 1994). Para la construcción de las matrices de similitud se utilizó el índice de Bray-Curtis (Somerfield, 2008). Los análisis estadísticos y gráficos fueron realizados con el programa estadístico "Past 3" (Hammer et al., 2001).

Dinámica poblacional: $A$. germinans domina en abundancia y extensión en el área de estudio (López-Portillo et al., 2014), por lo que se utilizó para la construcción de tablas de vida. Inicialmente, la tabla fue realizada con base en la supervivencia de la clase de plántula a juvenil, en todos los niveles de alteración (medida en el lapso de un año 2017 a 2018). Para la categoría de tamaños superiores se utilizaron las distribuciones de diámetros (DAP) para proyectar cambios en la población, como lo sugieren Condit et al. (1998) para poblaciones de árboles tropicales. La fecundidad fue estimada del trabajo de Cruz (2014), con base en la producción de propágulos de $A$. germinans en el área de estudio, para la temporada de máxima producción, de octubre a febrero.

La construcción de la tabla de vida se realizó con los criterios y formulación propuesta por Ali et al. (2008) para manglares del Golfo Arábigo, quienes asumen que la población se encuentra en una etapa demográfica estable, con insumos constantes y una distribución de edades estable, es decir, con producción anual de flores y propágulos y el reclutamiento de nuevos individuos a todos los estadios. Estos criterios se asumen en el presente estudio, debido a que es el primer trabajo para conocer la posible dinámica poblacional de $A$. germinans en los niveles de alteración, tomando como base la condición de un manglar no degradado. Los valores de abundancia de los individuos pertenecientes a cada estadio están representados por cada $100 \mathrm{~m}^{2}$ (0.01 ha).

\section{RESULTADOS}

La ordenación multidimensional no métrica tuvo un valor de estrés de 0.2 , lo que la hace una ordenación útil (Clarke 1992). La ordenación muestra (Fig. 2), las unidades de muestreo en un plano bidimensional agrupadas 


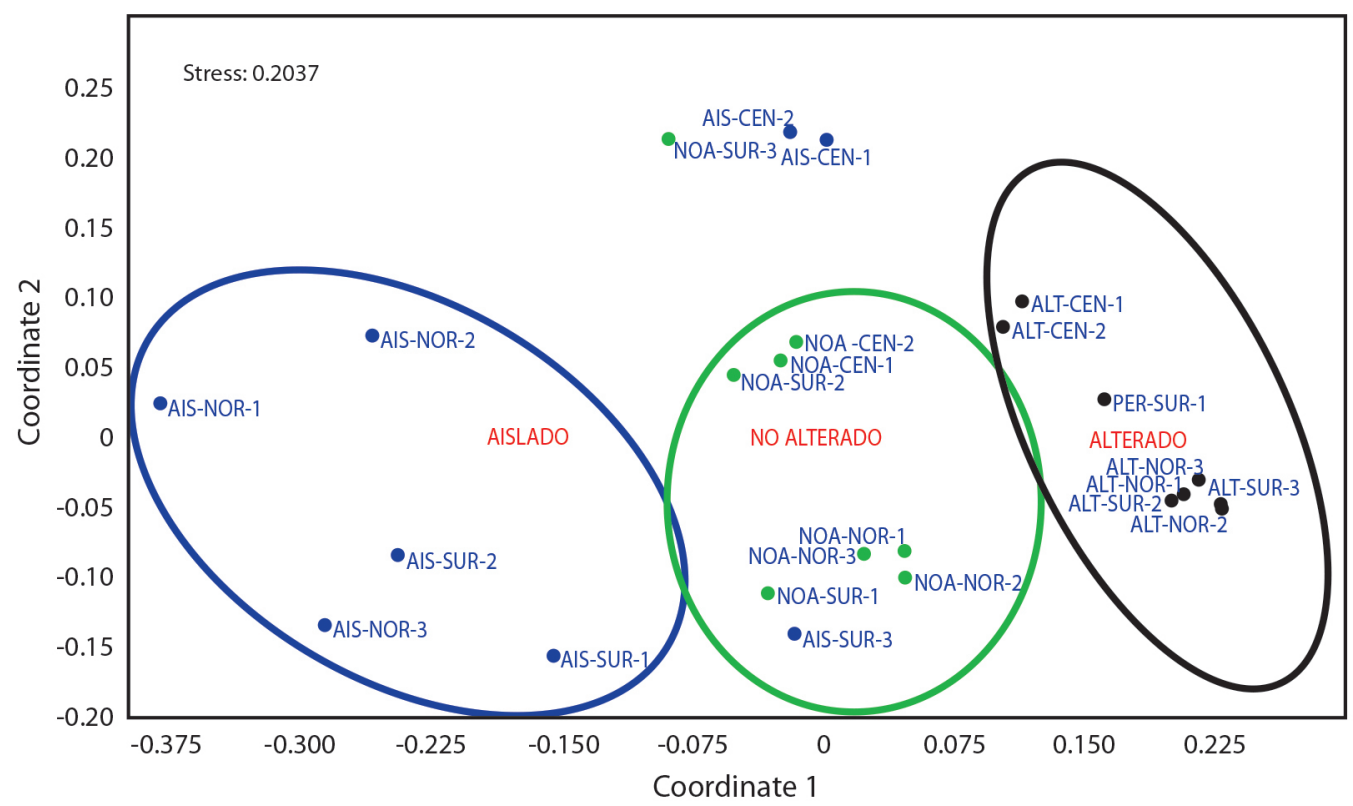

Fig. 2. Ordenamiento (nMDS) del muestreo realizado en el área de estudio por polígono (Z I, Z II y Z III), tipo de alteración del manglar (Aparentemente no alterado-NOA, Pérdida total o parcial de árboles-ALT y Aislado-AIS) con base en la abundancia de las clases de regeneración y estructura. Las tres figuras elípticas ejemplifican las ordenaciones por tipo: izquierda (azul) aislado, centro (verde), conservado, derecha (negro) mortalidad parcial.

Fig. 2. Ordination (nMDS) representing the samples at the study area by polygon (ZI, ZII and ZIII), the types of perturbation (apparently no perturbation (NO ALTERADO), perturbed (total or partial tree loss, ALTERADO) and isolated (AISLADO) based on the abundance of regeneration and structure stages. The ellipses show the samples by type: left (blue) isolated, center (green) no perturbation and right (black) partial mortality.

por tipo de alteración, a la izquierda, en el tipo aislado, las unidades de muestreo estuvieron representadas principalmente, por árboles adultos y maduros de L. racemosa; en el centro de la figura, el tipo no alterado, con ausencia de $L$. racemosa, con individuos de todas las categorías de tamaño de $A$. germinans y $R$. mangle; a la derecha, en el tipo alterado de la poligonal $\mathrm{Z}$ II estuvieron representadas todas las categorías de tamaño de $A$. germinans, excepto jóvenes, sin individuos de $R$. mangle y L. racemosa. Cabe señalar que en la parte superior de la figura se observan tres unidades de muestreo que coinciden en el mayor número de plántulas de A. germinans de sitios aislados y no alterados.

El análisis de similitudes (ANOSIM) mostró diferencias significativas entre los tres tipos de alteración $(\mathrm{P}<0.001, \mathrm{R}=0.59)$, con la prueba de valores de $\mathrm{p}$ corregidos con Bonferroni, todas las alteraciones fueron significativamente diferentes entre sí (no alterado-alterado $\mathrm{P}<$ 0.001 ; no alterado-aislado $\mathrm{P}<0.001$; y alterado-aislado $\mathrm{P}>0.001$ ).

Para conocer cuál de las clases de regeneración o estructura establece la disimilitud en los tipos de alteración, se aplicó una prueba de porcentaje de similitud (SIMPER) que mostró que la acumulación del $50 \%$ estaba influenciada, en primer lugar, por los árboles maduros de A. germinans, seguidos de las plántulas de la misma especie y posteriormente las plántulas de $R$. mangle, más abundantes en la zona conservada y aislada.

Las tablas de vida de $A$. germinans corresponden a cada una a un nivel de alteración. La supervivencia (lx) en el tipo no alterado (con 405 plántulas en el año inicial) muestra un descenso del $50 \%$ de esta categoría a la juvenil, 
pasando a 206 jóvenes (0.50), en tanto que la supervivencia del tipo alterado (100 plántulas iniciales) se reduce a $5 \%$ ( 5 plántulas), y en el tipo aislado (245 plántulas a 10 jóvenes) a un 4 $\%$. Para la siguiente categoría de regeneración, baja $15 \%$ en el sitio conservado, sube un 18 $\%$ en el alterado (de 5 jóvenes a 18 árboles jóvenes "a") y a $10 \%$ en el aislado (10 jóvenes a 25 árboles jóvenes "a"). Si bien se presenta un incremento en el número de árboles jóvenes "a" en los sitios alterado y aislado, no representa el número de éstos del sitio no alterado (18 y 25 contra 63, respectivamente). La supervivencia de la clase estructural árbol joven "b" baja a un individuo en todos los sitios, para incrementarse en los árboles maduros en 0.02 ( $2 \%$ ) en el sitio no alterado y a un $5 \%$ en el alterado, es decir, sin cambios en el sitio aislado. Sin embargo, en todos los sitios, las figuras típicas de supervivencia son de Tipo I o de una J invertida (Tabla 1, Tabla 2, Tabla 3).
Para la tasa de mortalidad (qx) por categoría, el comportamiento varía entre los tipos de alteración, en el tipo no alterado es de 0.49 (49\%) en plántulas, 0.69 (69\%) en jóvenes, 0.98 (98\%) en árboles jóvenes "a". Para las categorías de estructura, si bien la reducción en número es significativa, los individuos que llegan a la edad de 15 años en adelante tienen una tasa de mortalidad de cero e inclusive, se presenta una relación mayor de individuos adultos. En los sitios alterado y aislado se presenta una tasa de mortalidad alta en las plántulas $(0.95$ o $95 \%$ ), pero en los jóvenes ésta se revierte dado el incremento de individuos de joven a árbol joven "a", pero vuelve a caer a una proporción de 0.94 (94\%) en los árboles jóvenes "a", posteriormente se recupera en la categoría de adulto en el sitio alterado y se queda en cero en el aislado. El sitio no alterado tiene su etapa crítica solo en el estadio de árbol joven "a", sin embargo, los sitios alterado y aislado,

TABLA 1

Tabla de vida y fecundidad calculada para Avicennia germinans en sitios no alterados en la laguna de Tampamachoco, Veracruz, México

TABLE 1

Life table and fecundity calculated for Avicennia germinans at sites with no perturbation at the Tampamachoco lagoon, Veracruz, Mexico

\begin{tabular}{|c|c|c|c|c|c|c|c|c|c|c|c|c|}
\hline \multirow{2}{*}{ Estadios } & \multicolumn{12}{|c|}{ Aparentemente no alterado $\left(100 \mathrm{~m}^{2}\right)$} \\
\hline & $\mathrm{X}$ & $\mathrm{Nx}$ & $\mathrm{Lx}$ & $\mathrm{dx}$ & $\log 10 \mathrm{Nx}$ & $\mathrm{qx}$ & $\mathrm{Kx}$ & ex & bx & $1 \mathrm{xbx}$ & Xlxbx & $\mathrm{Vx}$ \\
\hline Plántula & 1 & 405 & 1.0000 & 0.4914 & 2.6075 & 0.4914 & 0.2936 & 1.6914 & & & & \\
\hline Juvenil & 2 & 206 & 0.5086 & 0.3531 & 2.3139 & 0.6942 & 0.5145 & 0.6914 & & & & \\
\hline árbol joven "a" & 6 & 63 & 0.1556 & 0.1531 & 1.7993 & 0.9841 & 1.7993 & 0.1827 & & & & \\
\hline Árbol joven "b" & 15 & 1 & 0.0025 & 0.0000 & 0.0000 & 0.0000 & 0.0000 & 0.0272 & 50 & 0.1234 & 1.8518 & 568.60 \\
\hline Árbol adulto & 25 & 1 & 0.0025 & -0.019 & 0.0000 & -8.000 & -0.954 & 0.0247 & 75 & 0.1851 & 4.6296 & 678.84 \\
\hline \multirow[t]{5}{*}{ Árbol maduro } & 35 & 9 & 0.0222 & 0.0000 & 0.9542 & 0.0000 & - & 0.0222 & 100 & 2.2222 & 77.7777 & 120.74 \\
\hline & & & & & & & & & & 2.5307 & 84.2592 & 1368.18 \\
\hline & Ro & 2.5309 & & & & & & & & & & \\
\hline & $\mathrm{T}$ & 33.2927 & & & & & & & & & & \\
\hline & $\mathrm{r}$ & 0.0282 & & & & & & & & & & \\
\hline
\end{tabular}

$\mathrm{X}=$ Edad estimada en años, $\mathrm{Nx}=$ Individuos por estadio, $\mathrm{Lx}=$ Proporción del cohorte original que sobreviven al comienzo de cada estadio, $\mathrm{dx}=$ Proporción del cohorte original que muere durante cada estadio, $\mathrm{qx}=$ Rango de mortalidad específica del estadio, $\mathrm{Kx}=$ Poder de mortalidad, $\mathrm{ex}=$ Expectativa de vida, $\mathrm{bx}=$ Número de propágulos por individuo, $\mathrm{Vx}=\mathrm{Rango}$ reproductivo, $\mathrm{T}=$ Tiempo generacional $\mathrm{y} \mathrm{r}=$ Rango de incremento intrínseco.

$\mathrm{X}=$ Estimated age in years, $\mathrm{Nx}=$ Number per stage, $\mathrm{Lx}=$ Proportion of original cohort surviving to start of each stage, $\mathrm{dx}=$ Proportion of original cohort dying during each stage, $\mathrm{qx}=$ Stage-specific mortality rate, $\mathrm{Kx}=$ Killing power, ex $=$ Expectation of future life, $b x=$ Average number of seeds per individual, $V x=$ Reproductive rate, $R=$ Net reproductive rate, $\mathrm{T}=$ Generation time, and $\mathrm{r}=$ Intrinsic rate of increase. 
TABLA 2

Tabla de vida y fecundidad calculada para Avicennia germinans en sitios alterados relacionados con la laguna de Tampamachoco, Veracruz, México

TABLE 2

Life table and fecundity calculated for Avicennia germinans at perturbated sites related to the Tampamachoco lagoon, Veracruz, Mexico

\begin{tabular}{|c|c|c|c|c|c|c|c|c|c|c|c|c|}
\hline \multirow{2}{*}{ Estadios } & \multicolumn{12}{|c|}{ Alterado $\left(100 \mathrm{~m}^{2}\right)$} \\
\hline & $\mathrm{X}$ & $\mathrm{Nx}$ & $\mathrm{Lx}$ & $\mathrm{dx}$ & $\log 10 \mathrm{Nx}$ & $\mathrm{qx}$ & $\mathrm{Kx}$ & ex & bx & $1 \mathrm{xbx}$ & Xlxbx & $\mathrm{Vx}$ \\
\hline Plántula & 1 & 100 & 1.0000 & 0.9500 & 2.0000 & 0.9500 & 1.3000 & 1.3000 & & & & \\
\hline Juvenil & 2 & 5 & 0.0500 & -0.1300 & 0.6990 & -2.6000 & -0.5563 & 0.3000 & & & & \\
\hline árbol joven "a" & 6 & 18 & 0.1800 & 0.1700 & 1.2553 & 0.9444 & 1.2553 & 0.2500 & & & & \\
\hline Árbol joven "b" & 15 & 1 & 0.0100 & 0.0000 & 0.0000 & 0.0000 & 0.0000 & 0.0700 & 7 & 0.07 & 1.05 & 89.26 \\
\hline Árbol adulto & 25 & 1 & 0.0100 & -0.0400 & 0.0000 & -4.0000 & -0.6990 & 0.0600 & 10 & 0.10 & 2.5 & 76.97 \\
\hline \multirow[t]{5}{*}{ Árbol maduro } & 35 & 5 & 0.0500 & 0.0000 & 0.6990 & 0.0000 & - & 0.0500 & 15 & 0.75 & 26.25 & 18.26 \\
\hline & & & & & & & & & & 0.92 & 29.80 & 184.49 \\
\hline & Ro & 0.9200 & & & & & & & & & & \\
\hline & $\mathrm{T}$ & 32.3913 & & & & & & & & & & \\
\hline & $\mathrm{r}$ & -0.0026 & & & & & & & & & & \\
\hline
\end{tabular}

$\mathrm{X}=$ Edad estimada en años, $\mathrm{Nx}=$ Individuos por estadio, $\mathrm{Lx}=$ Proporción de la cohorte original que sobreviven al comienzo de cada estadio, $\mathrm{dx}=$ Proporción del cohorte original que muere durante cada estadio, $\mathrm{qx}=$ Rango de mortalidad especifica del estadio, $\mathrm{Kx}=$ Poder de mortalidad, $\mathrm{ex}=$ Expectativa de vida, $\mathrm{bx}=$ Número de propágulos por individuo, $\mathrm{Vx}=\mathrm{Rango}$ reproductivo, $\mathrm{T}=$ Tiempo generacional $\mathrm{y} \mathrm{r}=$ Rango de incremento intrínseco.

$\mathrm{X}=$ Estimated age in years, $\mathrm{Nx}=$ Number per stage, $\mathrm{Lx}=$ Proportion of original cohort surviving to start of each stage, $\mathrm{dx}=$ Proportion of original cohort dying during each stage, $\mathrm{qx}=$ Stage-specific mortality rate, $\mathrm{Kx}=$ Killing power, ex $=$ Expectation of future life, $\mathrm{bx}=$ Average number of seeds per individual, $\mathrm{Vx}=$ Reproductive rate, $\mathrm{Ro}=$ Net reproductive rate, $\mathrm{T}=$ Generation time, and $\mathrm{r}=$ Intrinsic rate of increase.

TABLA 3

Tabla de vida y fecundidad calculada para Avicennia germinans en sitios aislados relacionados con la laguna de Tampamachoco, Veracruz, México

TABLE 3

Life table and fecundity calculated for Avicennia germinans at isolated sites related the Tampamachoco lagoon, Veracruz, Mexico

\begin{tabular}{|c|c|c|c|c|c|c|c|c|c|c|c|c|}
\hline \multirow{2}{*}{ Estadios } & \multicolumn{12}{|c|}{ Aislado $\left(100 \mathrm{~m}^{2}\right)$} \\
\hline & $\mathrm{X}$ & $\mathrm{Nx}$ & $\mathrm{Lx}$ & $\mathrm{dx}$ & $\log 10 \mathrm{Nx}$ & $\mathrm{qx}$ & $\mathrm{Kx}$ & ex & $\mathrm{bx}$ & $1 \mathrm{xbx}$ & Xlxbx & $\mathrm{Vx}$ \\
\hline Plántula & 0 & 245 & 1.0000 & 0.9592 & 2.3892 & 0.9592 & 1.3892 & 1.1551 & & & & \\
\hline Juvenil & 2 & 10 & 0.0408 & -0.061 & 1.0000 & -1.500 & -0.397 & 0.1551 & & & & \\
\hline árbol joven "a" & 4 & 25 & 0.1020 & 0.0980 & 1.3979 & 0.9600 & 1.3979 & 0.1143 & & & & \\
\hline Árbol joven "b" & 6 & 1 & 0.0041 & 0.0000 & 0.0000 & 0.0000 & 0.0000 & 0.0122 & 10 & 0.0408 & 0.6122 & 91.66 \\
\hline Árbol adulto & 15 & 1 & 0.0041 & 0.0000 & 0.0000 & 0.0000 & 0.0000 & 0.0082 & 15 & 0.0612 & 1.5306 & 35.96 \\
\hline \multirow[t]{5}{*}{ Árbol maduro } & 35 & 1 & 0.0041 & 0.0000 & 0.0000 & 0.0000 & - & 0.0041 & 20 & 0.0816 & 2.8571 & 31.40 \\
\hline & & & & & & & & & & 0.1836 & 4.9999 & 159.02 \\
\hline & Ro & 0.1837 & & & & & & & & & & \\
\hline & $\mathrm{T}$ & 27.2222 & & & & & & & & & & \\
\hline & $\mathrm{r}$ & -0.0623 & & & & & & & & & & \\
\hline
\end{tabular}

$\mathrm{X}=$ Edad estimada en años, $\mathrm{Nx}=$ Individuos por estadio, $\mathrm{Lx}=$ Proporción del cohorte original que sobreviven al comienzo de cada estadio, $\mathrm{dx}=$ Proporción del cohorte original que muere durante cada estadio, $\mathrm{qx}=$ Rango de mortalidad específica del estadio, $\mathrm{Kx}=$ Poder de mortalidad, $\mathrm{ex}=$ Expectativa de vida, $\mathrm{bx}=$ Número de propágulos por individuo, $\mathrm{Vx}=\mathrm{Rango}$ reproductivo, $\mathrm{T}=$ Tiempo generacional y $\mathrm{r}=$ Rango de incremento intrínseco.

$\mathrm{X}=$ Estimated age in years, $\mathrm{Nx}=$ Number per stage, $\mathrm{Lx}=$ Proportion of original cohort surviving to start of each stage, $\mathrm{dx}=$ Proportion of original cohort dying during each stage, $\mathrm{qx}=$ Stage-specific mortality rate, $\mathrm{Kx}=$ Killing power, ex $=$ Expectation of future life, $\mathrm{bx}=$ Average number of seeds per individual, $\mathrm{Vx}=$ Reproductive rate, $\mathrm{Ro}=$ Net reproductive rate, $\mathrm{T}=$ Generation time, and $\mathrm{r}=$ Intrinsic rate of increase. 
la presentan en las plántulas y en los árboles jóvenes "a".

La potencia de mortalidad $(\mathrm{Kx})$ es la proporción de la cohorte original que muere entre dos estadios. A la categoría de árbol joven "a" pertenecen los individuos que aún no alcanzan la madurez reproductiva (que se adquiere en los árboles jóvenes "b"), esta categoría muestra una potencia de mortalidad de 1.79 en el sitio no alterado, 1.25 en el alterado y 1.39 en el aislado. En los sitios alterado y aislado, se suma una potencia de mortalidad de plántulas de 1.30 y 1.38 , respectivamente. Lo que se observa es un solo pico de mortalidad en los sitios no alterado y dos picos en los sitios alterado y aislado.

El indicador que mejor explica la situación del sistema en general es la expectativa de vida (ex) de las clases de regeneración (plántula, juvenil y árbol joven "a"), es más alta en el sitio no alterado que en el resto de los sitios. En el sitio alterado se observa lo contrario, son bajas las expectativas de vida en las clases de regeneración, pero tiene los valores más altos en las clases estructurales (árbol joven "b", árbol adulto y árbol maduro). La expectativa de vida en todas las clases del sitio aislado son menores que en los otros dos sitios.

Aunque las abundancias son similares en las categorías de estructura del tipo no alterado con las presentes en el alterado y aislado, el número de individuos le otorga un rango reproductivo (lxbx) y un Rango Neto Reproductivo (R0) alto (2.53). El Tiempo Generacional (T) para los tres tipos de alteración es superior a los 25 años. El rango reproductivo del sitio no alterado es mayor que 1 , que significa crecimiento de la población, el sitio alterado tiene 0.92 , por debajo de 1 , con disminución y el aislado 0.18 , con una disminución poblacional mayor. El mismo patrón se observa en la Tasa Intrínseca de Aumento (r), con un valor bajo (0.0279) ligeramente mayor de cero (la población aumenta), los otros sitios presentan valores negativos $(-0.0026$ para el alterado y -0.0623 en el aislado), que significa que su población disminuye.

Por último, el valor reproductivo $(\mathrm{Vx})$ de la clase árbol joven "b" para el tipo no alterado es de 568.6, es decir, cada individuo dejará una descendencia de 568 nuevos individuos, el sitio alterado, por todo lo expresado anteriormente, solo tiene un valor reproductivo de 89.26 y el sitio aislado presenta un valor de 91.66. Al comparar las siguientes categorías estructurales (árbol adulto y maduro) se entiende mejor la dinámica poblacional de la especie por tipo de alteración para los árboles adultos, el tipo no alterado presenta un valor de 678.84, el alterado 76.97 el aislado 35.96, la siguiente clase de árbol maduro, el tipo no alterado presenta un valor reproductivo de 120.74 , en el alterado es de 18.26 y el aislado tiene un valor de 31.40 . El tipo no alterado, tiene dos clases con alto valor reproductivo, en contraste los sitios alterado y aislado, que tienen un bajo valor en la categoría árbol joven "b" y sigue su disminución en las otras dos clases.

\section{DISCUSIÓN}

La ordenación nMDS ha sido útil para describir la estructura poblacional de los bosques de mangle (Agudelo, 2000; Méndez \& Jiménez, 2015; Montes et al., 1999; Natividad et al., 2015; Satyanarayana et al., 2009). En este estudio se mostró que las áreas alteradas, determinadas a priori por diferencias visuales, corresponden a diferencias en la abundancia por especie bien definidas por sitio, no alterado, alterado y aislado. La zona no alterada se estructura con la presencia de todas las categorías de tamaño, excepto de la especie $L$. racemosa, más relacionada con el sitio aislado. El sitio aislado presenta un aspecto relevante, no presenta relación con el nivel de marea, ni con un flujo hidrológico interno debido a que es un talud de un terraplén construido para el canal de descarga de aguas tratadas del Complejo Termoeléctrico.

Aproximadamente el $25 \%$ de las plántulas establecidas son reclutadas al estadio juvenil en nichos de regeneración largos, si la alteración es pequeña cambia a un $10 \%$ (Clarke \& Allaway, 1993). La supervivencia reportada en los tipos alterado y aislado, presentaron el $0.05(5 \%)$ y 0.04 (4\%), lo que evidencia una 
alteración más bien grande, relacionado con un cambio de flujo hidrológico. Con relación a la mortalidad de la clase de plántulas, autores como Rabinowitz (1978), Clarke (1992), Delgado et al. (1999) y Ali et al. (2008), reportan una mortalidad alta $(>0.90)$, relacionada con la fase post-cotiledonaria, cuando la supervivencia ya depende de los recursos del medio en que se desarrollan (Clarke \& Allaway, 1993). Delgado et al. (1999), reportan un valor de 0.985 en el Pacífico de Costa Rica para Avicennia bicolor, valor muy próximo con el presentado en los sitios no alterado (0.95) y aislado (0.9592), en donde se presentan la mayor cantidad de plántulas.

De las plántulas que sobreviven, un porcentaje mayor al $50 \%$ logra pasar a la categoría de juveniles, sin embargo, para la categoría siguiente, árbol joven "a", el rango de mortalidad específica (qx) supera el valor de 0.95 en los tres sitios. Según Landsberg y Sands (2010), la supervivencia de esta categoría y las subsecuentes, dependerá de condiciones específicas, en áreas muy densas tiende a disminuir debido a competencia interespecífica $(0.0025$ en el sitio no alterado y 0.0041 en el aislado) $\mathrm{y}$, en áreas más abiertas, la supervivencia está en función de eventos que causan variaciones ( 0.01 en el tipo alterado). En los tipos no degradado y aislado es muy probable que se presente una competencia interespecífica muy alta, y en el tipo alterado debe estar influenciado por el efecto de la alteración. Se debe tomar en consideración que en el sitio alterado se contabilizaron las categorías $>2.5 \mathrm{~cm}$ de DAP, aun cuando era evidente el estrés de los individuos. El ajuste de esta supervivencia diferenciada entre sitios se hace evidente en el reporte del número de propágulos por individuo, el cual disminuye drásticamente en el sitio alterado.

Es en el estadio de árbol joven "b" la etapa en la que se observa la producción de propágulos viables, aproximadamente a los 15 años de edad, Clarke (1992) asigna esta etapa a los cinco años (árbol joven "a"), pero en condiciones de alta densidad o rápido crecimiento, la sitúa en los 20 años, estableciendo que el potencial de mayor fecundidad es en la madurez. En el trabajo realizado por Cruz (2014) se reporta un promedio de 74 propágulos por árbol en DAP mayores de $10 \mathrm{~cm}$, reduciendo su producción a medida que los DAP se hacen menores, como lo menciona Delgado et al. (1999) para A. bicolor.

Las categorías de plántula y árbol joven "a" en el tipo alterado, al presentar un poder de mortalidad (kx) mayor a 1, representan los estadios más débiles de $A$. germinans, en donde las fuerzas estresantes presentes están actuando. Este resultado coincide con lo reportado por Ali et al. (2008) para Avicennia marina y, reforzado con el argumento de Harper y White (1974), los autores argumentan que las primeras etapas de la regeneración son las más débiles en las plantas. Este aspecto y la cantidad de individuos de las categorías árbol joven "b" y adulto, el estrés presente en los árboles maduros y la baja contribución de su valor reproductivo (Vx) en todas las categorías (61.49 en promedio), disminuye la posibilidad de existencia futura de la población.

Por otra parte, el poder de mortalidad más bajo en plántulas y jóvenes en los sitios no alterado (0.2936 en plántulas y 0.5145 en jóvenes) y una alta producción de descendencia en las etapas reproductivas (455 en promedio), les otorga a estos sitios un incremento poblacional (Ro de 2.53 y r de 0.0279 contra Ro de 0.92 y $r$ de -0.0026 de los sitios alterados). Es decir, el área ocupada por los individuos no alterados, al estar inmersos en un ambiente más heterogéneo, y aparentemente todavía libre de estresores, permite tener una dinámica demográfica, tal como lo observa Bowers (1994).

Al quedar demostrado que la fragmentación, producto de la construcción de los terraplenes, no influyó en la caracterización de las clases de regeneración y estructura del bosque, sino más bien la alteración ocasionada, se estableció una dinámica poblacional distinta para cada uno de los tipos de alteración, las evidencias establecidas determinan la viabilidad del área aparentemente no alterada. Su área contigua de degradación parcial no tiene una buena condición para asegurar su continuidad, sobre todo por sus categorías de plántulas y árbol 
joven "a", la primera asegurando las demás clases y la segunda por ser antesala del comienzo de la etapa reproductiva. Por último, el tipo de población de $A$. germinans que quedó aislada entre el área completamente degradada y el cambio de nivel topográfico al final del bosque, tampoco presenta signos de continuidad poblacional, debido principalmente a la limitación de espacio y a su competencia interespecífica.

La conclusión del presente estudio se basa en la abundancia de las categorías de tamaño de cada especie en el área de estudio, identificando plenamente tres sitios diferenciados donde A. germinans presenta problemas en sus categorías de regeneración, una alta mortalidad de plántulas y en la etapa anterior a la contribución reproductiva (árbol joven "a") en los sitios alterado y aislado que, sumado a un bajo poder reproductivo, muestran una población en disminución. Por lo que se considera necesaria la intervención, en el área de estudio, para retirar o minimizar la causa del estrés. Con base en los valores reportados en el análisis y las comparaciones entre los sitios es posible determinar que, eliminado el factor estresante (interrupción del flujo hidrológico), todos los sitios tienen, aún, la capacidad necesaria para una regeneración natural.

Declaración de ética: los autores declaran que todos están de acuerdo con esta publicación y que han hecho aportes que justifican su autoría; que no hay conflicto de interés de ningún tipo; y que han cumplido con todos los requisitos y procedimientos éticos y legales pertinentes. Todas las fuentes de financiamiento se detallan plena y claramente en la sección de agradecimientos. El respectivo documento legal firmado se encuentra en los archivos de la revista.

\section{AGRADECIMIENTOS}

Un agradecimiento especial para las autoridades del Complejo Termoeléctrico "Presidente Adolfo López Mateos" de la Comisión Federal de Electricidad por permitir el acceso al área de manglar contigua a las instalaciones. También contribuyeron en los muestreos de campo, los Biólogos Marinos Jonathan Eduardo Rangel Méndez y Eduardo Taboada Pérez. Especial agradecimiento a los integrantes de los Cuerpos Académicos Manejo de Ambientes Marinos y Costeros y Ecosistemas Costeros de la Facultad de Ciencias Biológicas y Agropecuarias.

\section{RESUMEN}

Introducción: Los manglares están en declive en el mundo, conocer su estructura y regeneración en diferentes niveles de alteración puede ayudar a comprender la dinámica poblacional ante situaciones de estrés.

Objetivo: La meta principal del estudio fue analizar la estructura comunitaria y estimar la trayectoria de las poblaciones de Avicenia germinans en diferentes sitios de manglar que presentan tres niveles de alteración.

Métodos: Ocho transectos de aproximadamente $500 \mathrm{~m}$ de largo y colocados de manera perpendicular a la laguna de Tampamachoco, Veracruz, interceptaron tres niveles de alteración en los sitios estudiados del bosque de manglar. En cada transecto, cuadrantes de 10 x $10 \mathrm{~m}, 5$ x $5 \mathrm{~m}$, y 1 x $1 \mathrm{~m}$ sirvieron para contar todos los árboles de mangle por especie y estadio de madurez en 2017 y 2018.

Resultados: La especie más abundante fue: $A$. germinans seguida de Rhizophora mangle y Laguncularia racemosa. Con base en su abundancia, se encontraron diferencias significativas según los tres grados de alteración (AM $\mathrm{R}=0.6$, $\mathrm{P}<0.001$ ), ilustrado en un análisis de ordenación no métrico (nMDS). Una tabla de vida para $A$. germinans permitió un análisis poblacional que mostró una tasa intrínseca de incremento poblacional (r) negativa para los sitios que presentan alteración $(-0.0027 \mathrm{u}-0.0774)$ y positiva $(0.0289)$ en el sitio sin alteración aparente. La supervivencia del estadio de plántula a juvenil varia de $50 \%$ en el sitio no alterados a 5 y $4 \%$ en los otros dos sitios.

Conclusiones: Se necesitan medidas para incrementar la sobrevivencia de las plántulas y revertir la disminución de las trayectorias en los sitios con alteración, de otra forma el manglar tendrá una viabilidad reducida en el término medio.

Palabras clave: manglar; fragmentación; perturbación; tablas de vida; dinámica poblacional; categorías de tamaño, alteración.

\section{REFERENCIAS}

Agudelo, C. P. (2000). Estructura de los bosques de manglar del Departamento de Bolivar y su relación con algunos parámetros abióticos (Trabajo de Grado). Universidad de Bogotá Jorge Tadeo Lozano, Colombia. 
Ali, A., Alfarhan, A., Robinson, E., \& Aldjain, I. (2008). Pattern of survival and mortality of mangrove populations grown at Al-Jubail area (Saudi Arabia) of the Arabian Gulf. American Journal of Agricultural and Biological Sciences, 3(3), 610-616. https://doi. org/10.3844/ajabssp.2008.610.616

Awal, M. A. (2014a). Analysis of relationship between salinity and top-dying diseases of Sundri trees in Sundarbans, Bangladesh. Science Journal of Analytical Chemistry, 2(4), 29-40. https://doi.org/10.11648/j. sjac.20140204.11

Awal, M. A. (2014b). Analysis of soil parameters causing dieback in Sundarbans. American Journal of Environmental Protection, 3(3), 162-171. https://doi. org/10.11648/j.ajep.20140303.18

Basáñez-Muñoz, A. de J., Cruz, M. A., Domínguez, C., González, C., Serrano, A., \& Hernández, A. (2008). Estructura y producción de Conocarpus erectus L. en el Sitio Ramsar "Manglares y Humedales de Tuxpan”, Veracruz, México. Revista UDO Agrícola, 8(1), 78-87.

Basáñez-Muñoz, A. de J., Cuervo-López, L., Serrano-Solis, A., \& Cárdenas-del Ángel, S. I. (2016). Ensayos de reforestación con plantas de mangle en la Reserva Ecológica del Complejo Termoeléctrico "Presidente Adolfo López Mateos", Tuxpan, Veracruz. Polibotánica, 42, 91-101. https://doi.org/10.18387/ polibotanica. 42.5

Basáñez-Muñoz, A. de J., Olmedo, G., \& Rojas, P. (2006). Características estructurales y usos del manglar en el ejido Cerro de Tumilco, Tuxpan, Veracruz, México. Revista UDO Agrícola, 6(1), 114-120.

Bowers, M. A. (1994). Dynamics of age-and habitat structured populations. Oikos, 69, 327-333. https://doi. org/10.2307/3546154

Chen, G. C., Tam, N. F. Y., Wong, Y. S., \& Ye, Y. (2011) Effect of wastewaster discharge on greenhouse gas fluxes from mangrove soils. Atmosphreric Environment, 45, 1110-1115.

Chow, J. (2017). Mangrove management for climate change adaptation and sustainable development in coastal zones. Journal of Sustainable Forestry, 37(2), 139-156. https://doi.org/10.1080/10549811.2017.13 39615

Cintrón-Molero, G., \& Schaeffer-Novelli, Y. (1992). Ecology and management New World mangroves. In U. Seeliger (Ed.), Coastal plant communities of Latin America (pp. 233-258). Academic Press.

Clarke, P. J. (1992). Predispersal mortality and fecundity in the grey mangrove (Avicennia marina) in southeastern Australia. Australian Journal of Ecology, 17, 161-168. https://doi.org/10.1111/j.1442-9993.1992. tb00794.x
Clarke, P. J. (1995). The population dynamics of the mangrove Avicennia marina; demographic synthesis and predictive modelling. Hydrobiologia, 295, 83-88.

Clarke, P. J., \& Allaway, W. (1993). The regeneration niche of the grey mangrove (Avicennia marina); effects of salinity, light and sediment factors on establishment, growth and survival in the field. Oecologia, 93, 548-556. https://doi.org/10.1007/BF00328964

Clarke, K. R., \& Warwick, R. M. (1994). Ordination of samples by Multi-Dimensional Scaling (MDS). In K. R. Clark, \& R. M. Warwick (Eds.), Change in marine communities: an approach to statistical analysis and interpretation (pp. 1-12). PRIMER-E Ltd.

CONANP (Comisión Nacional de Áreas Naturales Protegidas). (2019). Humedales de México. http:// www.conanp.gob.mx/conanp/dominios/ramsar/docs/ sitios/FIR RAMSAR/Veracruz/Manglares $\% 20 \mathrm{y} \% 20$ Humedales\%20de \%20Tuxpan/Manglares\%20y\%20 Humedales\%20de\%20Tuxpan.pdf

Condit, R., Sukumar, R., Hubbell, S. P., \& Foster, R. (1998). Predicting population trends from size distributions: A direct test in a tropical tree community. The American Naturalist, 152(4), 495-509. https:// doi.org/10.1086/286186

Cruz, L. E. (2014). Capacidad reproductiva real de tres especies de mangle (Rhizophora mangle, Avicennia germinans $y$ Laguncularia racemosa) en la Reserva Ecológica del C.T.P.A.L.M (Tesis de Licenciatura). Universidad Veracruzana, México.

Delgado, P., Jiménez, J. A., \& Justic, D. (1999). Population dynamics of mangrove Avicennia bicolor on the Pacific coast of Costa Rica. Wetlands Ecology and Management, 7, 113-120.

Ewel, K. C., Twilley, R. R., \& Ong, J. E. (1998). Different Kinds of Mangrove Forests Provide Different Goods and Services. Global Ecology and Biogeography Letters, 7(1), 83-94. https://doi.org/10.2307/2997700

Giri, Ch., Long, J., Abbas, S., Murali, R. M., Qamer, F. M., Pengra, B., \& Thau, D. (2014). Distribution and dynamics of mangrove forests of South Asia. Journal of Environmental Management, 148, 101-111. https://doi.org/10.1016/j.jenvman.2014.01.020

Hamilton, S. E., \& Casey, D. (2016). Creation of a high spatio-temporal resolution global database of continuous mangrove forest cover for the 21 st century (CGMFC-21). Global Ecology and Biogeography, 25(6), 729-738. https://doi.org/10.1111/geb.12449

Hammer, Ø., Harper, D. A., \& Ryan, P. D. (2001). PAST: Paleontological statistics software package for education and data analysis. Palaeontologia Electronica, $4(1), 1-9$.

Harper, J. L., \& White, J. (1974). The demography of plants. Annual Review of Ecology and Systematic, 5, 419-463. https://doi.org/10.1146/annurev. es. 05.110174 .002223 
Islam, M., \& Bhulyan, M. A. H. (2108). Sundarbans mangrove forest of Bangladesh: causes of degradation and sustainable management options. Environmental Sustainability, 1, 113-131. https://doi.org/10.1007/ s42398-018-0018-y

Islam, M., Borgqvist, H., \& Kumar, L. (2019). Monitoring Mangrove forest landcover changes in the coastline of Bangladesh from 1976 to 2015. Geocarto International, 34(13), 1458-1476. https://doi.org/10.1080/1 0106049.2018 .1489423

Kairo, J. G., Dahdouh-Guebas, F., Gwada, P. O., Ochieng, C., \& Koedam, N. (2002). Regeneration status of mangrove forests in Mida Creek Kenya: a compromised or secured future? Ambio, 31(7-8), 562-568. https:// doi.org/10.1639/0044-7447(2002)031[0562:RSOMF I]2.0.CO;2

Landsberg, J. J., \& Sands, P. (2010). Stand structure and dynamics. In J. J. Landsber, \& P. Stands (Eds.), Physiological ecology of forest production (1 ${ }^{\text {era }}$ ed., Vol. 4, pp. 83-85). Academic Press.

López-Portillo, J. A., Lara, A. L., Sáinz, E., Vásquez, V. M., Rodríguez, M., Martínez, M. C., Bartolo, O., Ortiz, I. I., \& Alvarado, G. (2014). Restauración hidráulica en la laguna de Tampamachoco en el estado de Veracruz para la rehabilitación del manglar y de sus servicios ambientales. Instituto de Ecología A.C., México. Informe Final SNIB-CONABIO. Proyecto No. HH025.

Méndez, C., \& Jiménez, J. (2015). Análisis de la asociación entre la estructura y composición de la comunidad vegetal de manglar, y los parámetros físico-químicos del agua en la Bahía La Graciosa, Izabal, Guatemala. Revista Cientifica Instituto de Investigaciones Quimicas y Biológicas, 25(1), 50-64.

Montes, C. G., Castillo, S., \& López-Portillo, J. (1999) Distribución del manglar en cuatro sistemas lagunares en la costa de Chiapas, México. Boletín de la Sociedad Botánica de México, 64, 25-34. https://doi. org/10.17129/botsci.1579

Moreno, P., Rojas, J. L., Zarate, D., Ortiz, M. A., Lara, A. L., \& Saavedra, T. (2002). Diagnóstico de los manglares de Veracruz: distribución, vínculo con los recursos pesqueros y su problemática. Madera $y$ Bosques, 8(1), 61-88. https://doi.org/10.21829/ myb.2002.801292

Moreno-Casasola, P., Cejudo, E., Monroy, R. C., Infante, D. M., López, H., Peralta, L. A., Flores, I. N., Castillo-Campo, G., Madero, C., Capistrán, A., Rincón, M., \& Pérez, S. (2016). IV. Manglares, selvas inundables y humedales herbáceos. En P. Moreno-Casasola (Ed.), Servicios Ecosistémicos de las Selvas y Bosques costeros de Veracruz (pp. 76-93). INECOLITTO-CONAFOR-INECC, México.

Natividad, E. M. C., Hingabay, V. S., Lipae, H. B., Requieron, E. A., Abulunan, A. J., Tagaloguin, P. M., Flamiano, R. S., Jumawan, J. H., \& Jurnawan, J. C. (2015). Vegetation analysis and community structure of mangroves in Alabel and Maasim Sarangani Province, Philippines. ARPN Journal of Agricultural and Biological Science, 10(3), 97-102.

Osland, M. J., Day, R. H., Hall, C. T., Brumfield, M. D., Dugas, J. L., \& Jones, W. R. (2017). Mangrove expansion and contraction at a poleward range limit: climate extremes and land-ocean temperature gradients. Ecology, 98(1), 125-137. https://doi. org/10.1002/ecy. 1625

Owuor, M. A., Mulwa, R., Otieno, P., Icely, J., \& Newton, A. (2019). Valuing mangrove biodiversity and ecosystem services: A deliberative choice experiment in Mida Creek, Kenya. Ecosystem Services, 40, 101040. https://doi.org/10.1016/j.ecoser.2019.101040

Rabinowitz, D. (1978). Mortality and initial propagule size in mangrove seedlings in Panama. Journal of Ecology, 66, 45-51.

Rahman, M. A. (1994). Final report on mangrove plant pathology of the Sundarbans reserved forest in Bangladesh. FAO; UNDP (Eds.), Integrated Resources Development of the Sundarbans Reserved Forest (Field document 3, pp. 1-81). Khulna.

Sasmito, S. D., Murdiyarso, D., Friess, D. A., \& Kurnianto, S. (2015). Can mangroves keep pace with contemporary sea level rise? A global data review. Wetlands Ecology and Management, 23(5), 263-278. https:// doi.org/10.1007/s11273-015-9466-7

Satyanarayana, B., Raman, A. V., Mohd-Lokman, H., Dehairs, F., Sharma, V. S., \& Dahdouh-Guebas, F. (2009). Multivariate methods distinguishing mangrove community structure of Coringa in the Godavari Delta, East coast of India. Acuatic Ecosystem Heath \& Management, 12(4), 401-408. https://doi. org/10.1080/14634980903334074

Somerfield, P. J. (2008). Identification of the Bray-Curtis similarity index: Comment on Yoshioka. Marine Ecology Progress Series, 372, 303-306. https://doi. org/10.3354/meps07841

Valderrama-Landeros, L. H., Rodríguez-Zúñiga, M. T., Troche-Souza, C., Velázquez-Salazar, S., VilledaChávez, E., Alcántara-Maya, J. A., Vázquez-Balderas, B., Cruz-López, M. I., \& Ressl, R. (2017). Manglares de México: actualización y exploración de los datos del sistema de monitoreo 1970/1980-2015. Comisión Nacional para el Conocimiento y Uso de la Biodiversidad (CONABIO), México.

Vovides, A. G., López-Portillo, J., \& Bashan, Y. (2011). N2-fixation along a gradient of long-term disturbance in tropical mangroves bordering the Gulf of Mexico. Biology and Fertiility of Soils, 47(5), 567-576. https://doi.org/10.1007/s00374-011-0562-4

Warwick, R. M., \& Clarke, K. R. (1991). A comparison of some methods for analysing changes in benthic community structure. Journal of the Marine Biological Association of the United Kingdom, 71(1), 225-244. https://doi.org/10.1017/S0025315400037528 